\title{
Comparative Spectrophotometer Analysis of Ultraviolet-light Filtering, Blue-light Filtering, and Violet-light Filtering Intraocular Lenses
}

\author{
Jeong Woo Park, Chul Young Choi \\ Department of Ophthalmology, Kangbuk Samsung Hospital, Sungkyunkwan University School of Medicine, Seoul, Korea
}

\begin{abstract}
Purpose: To compare the light transmittance property of seven currently used intraocular lens (IOL) models by spectrophotometer data.

Methods: Light transmission spectra of seven IOL models were assessed with a spectrophotometer. The transmittance properties were analyzed in $1 \mathrm{~nm}$ units from $350 \mathrm{~nm}$ wavelength to $800 \mathrm{~nm}$.

Results: Three ultraviolet filtering IOL models (ZCB00, XC1-SP, and AT LISA 809M) showed nearly full transmittance of the light from 400 to $500 \mathrm{~nm}$, while steeply attenuating light with shorter wavelengths in various degrees. Three blue-light filtering IOLs (yellow-tinted IOLs; XY1, SN60WF, and TNFT00) showed a slow-sloped increase of light transmission between 400 to $500 \mathrm{~nm}$. Among the three, XY1 showed different degree of inclination, showing a steeper slope than SN60WF and TNFT00. The violet-light filtering IOL (ZFROOV) showed a rapid increase of the transmission at around $435 \mathrm{~nm}$ wavelength, which is similar to ultraviolet filtering IOLs.

Conclusions: The seven different IOLs measured showed different characteristics of light transmission depending on the properties of each material and color. Blue-light filtering IOLs tend to blocked a wide range of wavelength up to $500 \mathrm{~nm}$, but rather were not effective at the range of 400 to $430 \mathrm{~nm}$. Violet-light filtering IOL showed advantages in filtering the high-energy wavelength, around $430 \mathrm{~nm}$, having a potential risk to retina and allowing the transmission of useful blue and green wavelength which is necessary for a better scotopic contrast sensitivity.
\end{abstract}

Key Words: Blue-light filtering intraocular lens, Spectrophotometer, Violet-light filtering intraocular lens

The human crystalline lens has a functional role in protecting the retina from ultraviolet (UV) phototoxicity apart from accommodation. UV light, which has a shorter wave-

Received: October 8, 2021 Final revision: October 9, 2021

Accepted: October 12, 2021

Corresponding Author: Chul Young Choi, MD, PhD. Department of Ophthalmology, Kangbuk Samsung Hospital, Sungkyunkwan University School of Medicine, 29 Saemunan-ro, Jongno-gu, Seoul 03181, Korea. Tel: 82-2-2001-2250, Fax: 82-2-2001-2262, E-mail: sashimi0@naver.com length than visible light, can be responsible for phototoxicity in retinal photoreceptors and pigment epithelial cells and can thus induce oxidative stress and damage [1,2]. UV light with wavelengths of shorter than $300 \mathrm{~nm}$ is mostly absorbed in the cornea [3], whereas UV light from 300 to $400 \mathrm{~nm}$ is usually absorbed in the crystalline lens depending on the degree of cataract. To prevent the retina from phototoxicity after cataract surgery, intraocular lenses (IOLs) with UV-light blocking properties are widely used in the clinic [4]. 
Blue light is a type of high-energy visible (HEV) light with wavelengths ranging from 400 to $500 \mathrm{~nm}$. Under scotopic conditions, human eyes are known to see light with such wavelengths with the help of the rod cells' sensitivity at this range of wavelengths [5]. In addition, the blue light also plays an important role in human circadian rhythm by affecting melatonin secretion of the pineal gland [6]. According to the latest research, blue light may, just as UV light, also cause phototoxicity in retinal photoreceptors and the pigment epithelium [2]. Various studies on the efficacy of blue light attenuation have been conducted, because light emitting diode light sources, which have been widely adopted in recent years, emit a lot of blue light [1,7-9]. In general, HEV light, often called "blue light," is divided into the 400 to $440 \mathrm{~nm}$ violet spectrum and the 440 to 500 $\mathrm{nm}$ blue spectrum [9,10]. Of all blue light, the violet spectrum is known to cause more retinal phototoxicity while it has less effect on scotopic vision, contrast sensitivity, and circadian rhythm $[4,7]$. Therefore, there might be a need of IOLs that protect against the specific violet-light spectrum. In this study, several UV-filtering IOLs, UV- and blue-light filtering yellow-tinted IOLs, and the violet-light filtering IOL, which are widely used in clinical practice, are analyzed and compared in terms of light transmittance using a spectrophotometer.

\section{Materials and Methods}

A total of seven IOL models were assessed with a spectrophotometer (Cary 5000 UV/VIS/NIR; Agilent technologies, Santa Clara, CA, USA), with high accuracy in wavelength analysis from UV wavelength $(175 \mathrm{~nm})$ to near-infrared $(3,300 \mathrm{~nm})$. The IOLs, a violet-light filter (Synergy ZFR00V; Johnson \& Johnson, New Brunswick, NJ, USA), a UV-light filter (Tecnis ZCB00, Johnson \& Johnson; XC1-SP, Hoya, Tokyo, Japan; and AT LISA 809M, Carl Zeiss, Jena, Germany), or a blue-light filter (yellow-tinted IOLs; Acrysof IQ SN60WF; PanOptix TNFT00; Alcon, Geneva, Switzerland; XY1, Hoya) were evaluated (Fig. 1). All the power of IOLs tested were 20.0 diopters to minimize experimental errors possibly due to a dioptric difference. Transparent glass panel was prepared for every IOL specimens. Each IOL's haptics were fixed on transparent dry glass panel with optic position in parallel with the glass panel for spectrophotometry. The IOL speci- mens were then positioned under the spectrophotometer's measuring beam with IOL's optic center positioned to match the center of the reference beam. Each specimen was tested for its light transmission intensity (in \%) and repeated five times. To eliminate confounding effect of panel glass, baseline transmission data with and without panel glass were measured and deducted from the specimens' value. Then the mean values of all IOLs' light transmission intensity were calculated for each wavelength in 1 $\mathrm{nm}$ units from 350 to $800 \mathrm{~nm}$. The averaged values were displayed in a representative transmission curve.

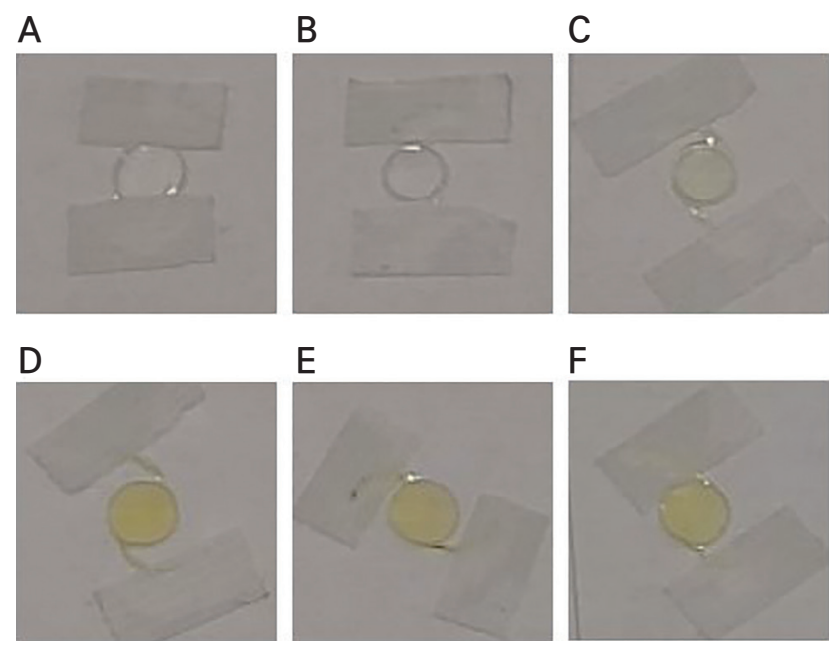

Fig. 1. Gross photograph of various intraocular lenses and transparent glass plates used in this experiment. (A) AT LISA $809 \mathrm{M}$, (B) ZCB00, (C) ZFR00V, (D) TNFT00, (E) SN60WF, and (F) $\mathrm{XY}-1$.

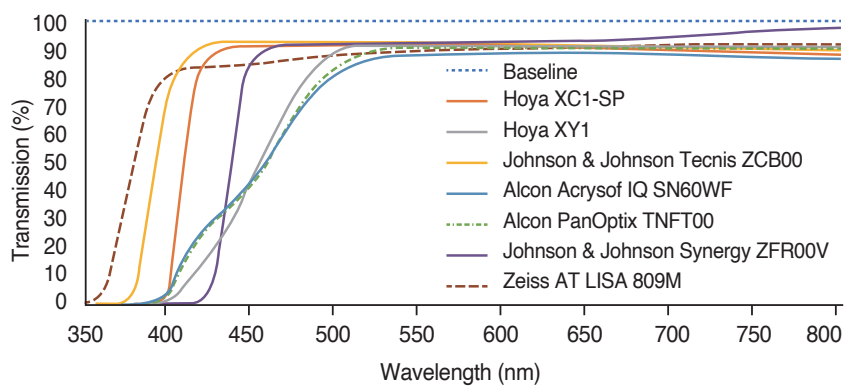

Fig. 2. Transmission spectra of various intraocular lenses (IOLs). Data of yellow-tinted IOLs (blue-light filtering IOLs) tends to gradually increase between 400 and $500 \mathrm{~nm}$ while transmission in ultraviolet-light filtering IOLs drastically increases at certain wavelengths of less than $400 \mathrm{~nm}$. The violet-light filtering IOL shows a graph similar to that of ultraviolet-light filtering IOLs, but the inflection point is at around $435 \mathrm{~nm}$. 
Table 1. Spectrophotometer data of various IOLs at different wavelengths

\begin{tabular}{lcrcccc}
\hline Variable & $375 \mathrm{~nm}$ & $400 \mathrm{~nm}$ & $425 \mathrm{~nm}$ & $450 \mathrm{~nm}$ & $475 \mathrm{~nm}$ & $500 \mathrm{~nm}$ \\
\hline Hoya XC1-SP & $0.00 \pm 0.00$ & $7.20 \pm 0.01$ & $88.46 \pm 0.05$ & $91.41 \pm 0.06$ & $91.75 \pm 0.06$ & $91.89 \pm 0.06$ \\
Zeiss LISA 809M & $40.8 \pm 0.02$ & $80.24 \pm 0.05$ & $83.63 \pm 0.04$ & $84.65 \pm 0.05$ & $86.43 \pm 0.06$ & $87.74 \pm 0.04$ \\
Tecnis ZCB00 & $0.10 \pm 0.00$ & $73.40 \pm 0.03$ & $91.31 \pm 0.01$ & $92.45 \pm 0.02$ & $92.43 \pm 0.02$ & $92.39 \pm 0.03$ \\
Synergy ZFR00V & $0.00 \pm 0.00$ & $0.00 \pm 0.00$ & $6.23 \pm 0.02$ & $86.05 \pm 0.02$ & $91.51 \pm 0.02$ & $91.85 \pm 0.02$ \\
Hoya XY1 & $0.00 \pm 0.00$ & $0.93 \pm 0.01$ & $18.44 \pm 0.01$ & $45.65 \pm 0.02$ & $73.95 \pm 0.02$ & $88.81 \pm 0.03$ \\
Alcon SN60WF & $0.00 \pm 0.00$ & $4.64 \pm 0.02$ & $28.67 \pm 0.11$ & $44.68 \pm 0.10$ & $66.29 \pm 0.04$ & $81.28 \pm 0.03$ \\
Alcon PanOptix & $0.00 \pm 0.00$ & $3.74 \pm 0.02$ & $27.70 \pm 0.13$ & $44.33 \pm 0.10$ & $67.53 \pm 0.02$ & $83.86 \pm 0.04$ \\
\hline
\end{tabular}

Values are presented as mean \pm standard deviation. Ultraviolet-light filtering IOLs show a plateau after $425 \mathrm{~nm}$ while blue-light filtering IOLs show a gradual increase of transmission between 400 and $475 \mathrm{~nm}$. The violet-light filtering IOL (ZFR00V) shows a rapid increase of transmission between 425 and $450 \mathrm{~nm}$ while absolutely blocking shorter wavelengths.

$\mathrm{IOL}=$ intraocular lens.

\section{Results}

All UV-light filtering IOLs (ZCB00, XC1-SP, and AT LISA) showed an excellent absorption within the UV-light spectrum with wavelengths shorter than $400 \mathrm{mn}$. However, these IOLs showed no attenuation in the HEV light spectrum which is part of the visible light spectrum with wavelengths greater than $400 \mathrm{~nm}$ (Fig 2.).

Likewise, the yellow-tinted UV- and blue-light filtering IOLs (SN60WF, TNFT00, and XY1), showed a full protection from the UV-light (wavelengths shorter than $400 \mathrm{~nm}$ ). In the HEV light spectrum between 400 and $500 \mathrm{~nm}$ attenuations were achieved. In that range, the protection of the violet spectrum was higher than that of the blue spectrum.

The violet-light filter IOL (ZFR00V) steeply attenuated light with wavelengths under $430 \mathrm{~nm}$ compared to bluelight filtering IOLs and transmitted nearly all light with wavelengths of $440 \mathrm{~nm}$ or greater. The UV- and blue-light absorbing IOLs showed slow slope light-attenuating characteristics at wavelengths in the HEV light spectrum, whereas the violet-light filtering IOL and the UV-light filtering IOLs showed a tendency to rapidly increase the transmission at certain wavelengths (Table 1).

\section{Discussion}

In general, the wavelength of visible light is ranging from 400 to $700 \mathrm{~nm}$ and UV light is the range shorter than that. In recent studies, it has been discovered that HEV light with wavelengths ranging from 400 to $440 \mathrm{~nm}$ is potentially phototoxic to the retinal pigment epithelium of the retina, eventually which could lead to macular degeneration $[2,8,10]$. Due to the normal ageing process of the human crystalline lens, which gradually attenuates HEV light, the retina is protected from phototoxicity in midaged and older patients. In pseudophakic eyes, the macula can be exposed to retinal toxicity when all the wavelengths of the visible spectrum and ultraviolet light are transmitted [11].

A UV filtering function has been added to the majority of IOLs since 1980s. Later on, IOLs with a HEV light attenuation function have started to come out as many studies showed that HEV light may also have retinal toxicity. However, these yellow-tinted IOLs can have negative effects on patients' contrast sensitivity, as they partially attenuate light within the visible spectrum. Especially the rod cell-mediated scotopic and mesopic vision relies also on the blue-light spectrum [7], Nakano et al. [4] compared surgery results with blue light-filtering IOLs and violet-light filtering IOLs. Patients who had received a violet-light filtering IOL achieved 3 months after surgery better results in terms of contrast sensitivity under photopic and mesopic conditions when compared with patients who had received blue light-filtering IOLs [4].

Furthermore, in natural environments, blue light has an inhibitory effect on melatonin secretion through a retinal melanopsin reaction by the pineal gland. Thus, during night-time with less blue light, melatonin secretion increases, which makes people sleepy. Therefore, blue light 
is involved in the human circadian rhythm. When such blue light is intentionally blocked, it can be expected to have a negative effect on the sleep cycle. However, in their study on a correlation between blue-light filtering IOLs and sleep quality, Feng et al. [6] discovered that there was no significant difference between patient groups who had received UV-light filtering IOLs or UV- and blue-light filtering IOLs. Both groups had better sleep quality after the surgery.

Violet-filtering IOLs specifically block HEV light with wavelengths shorter than $440 \mathrm{~nm}$. This is different from the concept of blue-light filtering IOLs that broadly attenuate light with wavelengths between 400 and $500 \mathrm{~nm}$. One of the main advantages of the violet-light filtering IOLs compared to blue-light filtering IOLs might be that they have less influence on light with wavelengths around 498 $\mathrm{nm}$ and improved scotopic vision can be expected because this could be the peak area of the rod cells' activity [5,9]. However, this has not yet been demonstrated in an in vivo study.

In this study, we compared the three different blue-filtering IOLs. Two IOLs (SN60WF and TNFT00) were made of same material, having different optical properties, and XY1 was made of different material. Interestingly there was a difference in the characteristics of transmission of light with different materials. XY1 showed a much steeper curve in the 400 to $500 \mathrm{~nm}$ range, suggesting slightly better filtering profile in the range of 400 to 430 $\mathrm{nm}$ than SN60WF and TNFT00. Three blue-filtering IOLs did not show the complete filtering HEV light in the range of 400 to $430 \mathrm{~nm}$ and they did not allow full transmittance of blue and green light.

Violet-filtering IOL in this study showed much steeper curve than blue-filtering IOLs and it is quite similar degree of inclination to UV filtering IOLs. It was very effective in blocking the wavelength less than $430 \mathrm{~nm}$ and did not interfere with the transmission of light from $450 \mathrm{~nm}$ or more. So in real life situation after cataract surgery with this type of IOLs, we can expect more effective blocking of HEV light to retina and less interference of healthy blue and green light which is known to be necessary for physiologic condition and contrast sensitivity in scotopic vision.

The limitation of this study in terms of experiment settings were, we tested all the IOLs in dry condition and one of the tested IOLs showed the result of transmittance of UV light between 360 to $380 \mathrm{~nm}$ whereas all the others not. The only different characteristic of the IOL beside the color was hydrophilic nature, and tested that IOL in airdried condition, so we need further testes of all models in same moisture chamber condition to compare each other.

In this study, violet-light filtering IOL showed a better transmittance characteristic, potential advantage to block high-energy wavelength which is known to have risk of reactive oxygen species damage to retinal pigment epithelium cells and allowing full transmission of healthy blue light compared to UV- and blue-light filtering IOLs. And we need further investigations in clinical aspect to confirm the violet-blocking IOLs can have such a superiority of contrast sensitivity in scotopic condition, circadian rhythms and sleep quality.

\section{Conflicts of Interest}

No potential conflict of interest relevant to this article was reported.

\section{Acknowledgements}

None.

\section{Funding}

The authors received no financial support for this article.

\section{References}

1. Downie LE, Busija L, Keller PR. Blue-light filtering intraocular lenses (IOLs) for protecting macular health. Cochrane Database Syst Rev 2018;5:CD011977.

2. Marie M, Bigot K, Angebault C, et al. Light action spectrum on oxidative stress and mitochondrial damage in A2E-loaded retinal pigment epithelium cells. Cell Death Dis 2018;9:287.

3. Doutch JJ, Quantock AJ, Joyce NC, Meek KM. Ultraviolet light transmission through the human corneal stroma is reduced in the periphery. Biophys $J$ 2012;102:1258-64.

4. Nakano S, Miyata A, Kizawa J, et al. Blue light-filtering 
and violet light-filtering hydrophobic acrylic foldable intraocular lenses: Intraindividual comparison. J Cataract Refract Surg 2019;45:1393-7.

5. Griswold MS, Stark WS. Scotopic spectral sensitivity of phakic and aphakic observers extending into the near ultraviolet. Vision Res 1992;32:1739-43.

6. Feng X, Xu K, Hao Y, Qi H. Impact of blue-light filtering intraocular lens implantation on the quality of sleep in patients after cataract surgery. Medicine (Baltimore) 2016;95:e5648.

7. Mainster MA. Intraocular lenses should block UV radia- tion and violet but not blue light. Arch Ophthalmol 2005;123:550-5.

8. Mainster MA, Sparrow JR. How much blue light should an IOL transmit? Br J Ophthalmol 2003;87:1523-9.

9. Mainster MA, Turner PL. Blue-blocking IOLs decrease photoreception without providing significant photoprotection. Surv Ophthalmol 2010;55:272-89.

10. Glickman RD. Phototoxicity to the retina: mechanisms of damage. Int J Toxicol 2002;21:473-90.

11. Mellerio J. Yellowing of the human lens: nuclear and cortical contributions. Vision Res 1987;27:1581-7. 